\title{
Health effects of vitamin and mineral supplements
}

\section{Growing numbers of healthy people are taking dietary supplements but there is little evidence that they protect against non-communicable diseases, say Fang Fang Zhang and colleagues}

\section{V} itamin and mineral supplements are the most commonly used dietary supplements by populations worldwide. ${ }^{1-4}$ The amount of micronutrients they provide ranges from less than recommended intakes to much more, making them important contributors to total intakes. While supplements can be used to correct micronutrient deficiency or maintain an adequate intake, over-the-counter supplements are most often taken by people with no clinical signs or symptoms of deficiency. However, the effect of vitamin and mineral supplements on the risk of non-communicable diseases in "generally healthy" populations is controversial. We examine patterns of supplement use and the evidence on their effects from randomised trials.

\section{Who uses supplements?}

Vitamin and mineral supplements have a large worldwide market, but we will focus on their use in North America and Europe, where there is most evidence on patterns of use and health outcomes. The use of vitamin, mineral, and fish oil supplements ${ }^{5}$ is common among adults in North America

\section{KEY MESSAGES}

- Randomised trial evidence does not support use of vitamin, mineral, and fish oil supplements to reduce the risk of non-communicable diseases

- People using supplements tend to be older, female, and have higher education, income, and healthier lifestyles than people who do not use them

- Use of supplements appreciably reduces the prevalence of inadequate intake for most nutrients but also increases the prevalence of excess intake for some nutrients

- Further research is needed to assess the long term effects of supplements on the health of the general population and in individuals with specific nutritional needs, including those from low and middle income countries (fig 1). ${ }^{6}$ The prevalence of use has increased for some individual nutrients-for example, there was a fourfold increase in use of vitamin D supplements among US adults from 1999 to 2012, excluding intake obtained from multivitamin and mineral. ${ }^{7}$ The use of omega-3 fatty acid supplements also increased sevenfold. ${ }^{7}$

Supplement use is generally less prevalent in other countries than in the US and Canada but varies widely (eg, Denmark $51 \%$, South Korea 34\%, Australia 43\%, UK $36 \%$, Spain 6\%, Greece 2\%). ${ }^{2-4}$ Different methods for assessing supplement use may contribute to the different prevalence in high income countries. National survey data for supplement use in the general population remain scarce for low and middle income countries.

Supplement use varies considerably among population subgroups within North America and Europe. In the US, $>70 \%$ of adults aged $\geq 65$ years use supplements ${ }^{8}$ compared with a third of children and adolescents. ${ }^{9}$ More women than men use supplements. ${ }^{6}$ Supplement use correlates positively with educational and socioeconomic status. ${ }^{10}$ It also clusters with healthy lifestyle factors such as not being a smoker or heavy drinker, not being overweight or obese, and being physically active. ${ }^{6}$ Importantly, people who use supplements tend to have a better overall diet quality than those who don't use them and their nutrient intake from foods mostly meets recommended intake levels ${ }^{.112}$

\section{Are supplements needed?}

Use of supplements contributes substantially to total vitamin and mineral intakes at the population level. ${ }^{13}$ Intake of vitamin $\mathrm{B}_{6}$, thiamin, and riboflavin among US adults is at least five times higher from supplements than from foods, and intakes are 15 to 20 times higher for supplements for vitamins $\mathrm{B}_{12}$ and $\mathrm{E} .{ }^{6}$ Consequently, supplement use considerably reduces the proportion of the general population with inadequate nutrient intake (box 1).

This is especially true for vitamins and minerals identified as "shortfall” nutrients such as calcium and vitamin D (fig 2). ${ }^{14}$ Despite the high use of supplements, inadequate intakes of micronutrients are still common in high income countries, where dietary patterns are typically energy rich but nutrient poor.

In low and middle income countries, where specific micronutrient deficiencies are prevalent (eg, of iodine, iron, zinc, and vitamin A), supplementation is recommended when food based approaches such as dietary modification, fortification, or food provision are unable to achieve inadequate intake. ${ }^{15}$ In the US and other countries, food fortification and enrichment such as the addition of iodine to salt, vitamin $D$ to milk, and $B_{1}$ and $B_{3}$ vitamins to refined flour have contributed to the virtual elimination of their syndromes of deficiency (goitre, rickets, beriberi, and pellagra, respectively). ${ }^{1617}$

The widespread use of vitamin and mineral supplements in high income countries seems to contribute to an increase in population prevalence of intake above the upper tolerable level (box 1). ${ }^{6}$ Although the overall proportion of US adults with intakes above the upper level is below 5\% for most nutrients (fig 2), some population subgroups may have high rates of excess intake. For example, in a Canadian national survey, over $80 \%$ of children aged 1-3 years who took dietary supplements consumed vitamin A and niacin at levels above the upper limit. ${ }^{18}$ In the US, excessive intake was noted for vitamin A (97\%) and zinc (68\%) among toddlers who were given supplements. ${ }^{19}$ High quality evidence is lacking on the long term adverse effects of excess intake for several nutrients so it is unclear whether this is a cause for concern.

\section{Do supplements protect against non- communicable diseases?}

It remains controversial whether supplements are effective in reducing the risk of non-communicable diseases. In contrast to results of observational studies, the accumulated evidence from randomised controlled trials does not support benefits of 


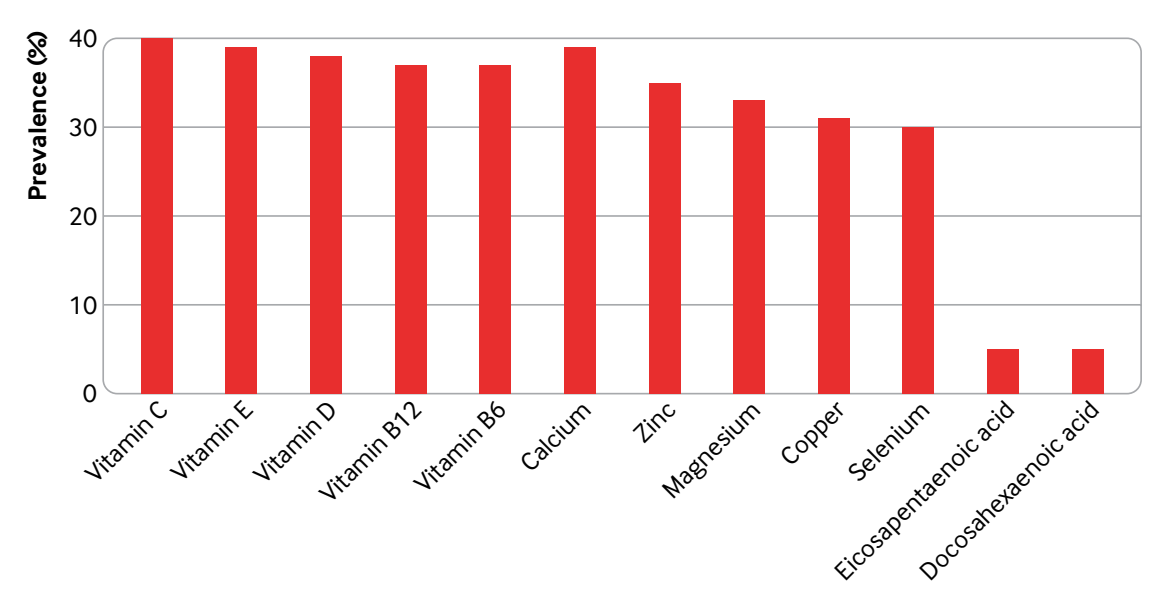

Fig 1 | Proportion of US adults taking commonly vitamin, mineral, and fish oil supplements, National Health and Nutrition Examination Survey 1999-2014 ${ }^{6}$

supplements in reducing risks of cardiovascular disease, cancer, or type 2 diabetes in healthy people with no clinical nutritional deficiencies.

\section{Cardiovascular disease}

An updated systematic review of 15 randomised trials published after the 2013 US Preventive Service Task Force (USPSTF) review ${ }^{20}$ confirmed the lack of benefits of supplements on cardiovascular events, mostly among patients with risk factors. ${ }^{21}$ Although randomised trials of folic acid, alone or in combination with vitamins $B_{12}$ or $B_{6}$, found significant reductions in plasma homocysteine levels, total cardiovascular events were not reduced. Another systematic review reported a reduced risk of stroke in association with supplementation of homocysteine lowering B vitamins, ${ }^{22}$ but the result was largely driven by one large trial in China. ${ }^{23}$ Overall, there is no consistent evidence to support the use of antioxidant supplements for reducing cardiovascular risk. ${ }^{2224}$

The Vitamin D and Omega-3 Trial (VITAL), one of the few randomised trials of supplements for primary prevention of cardiovascular disease, found no effect of vitamin D supplementation (2000 IU/ day) on its primary endpoint (myocardial infarction, stroke, or cardiovascular death) in healthy people. ${ }^{25}$ Previous large scale trials such as the Women's Health Initiative Calcium and Vitamin D Supplementation Study $^{26}$ and the Vitamin D Assessment Study ${ }^{27}$ also showed vitamin D supplements, alone or in combination with calcium, had no effect on cardiovascular risk.

Supplementation with omega-3 fatty acids ( $1 \mathrm{~g} /$ day) did not reduce the risk of major cardiovascular events among healthy people in the VITAL trial. ${ }^{28}$ However, benefits were found for some secondary endpoints such as total myocardial infarctions. This result is largely consistent with findings from metaanalyses that fish oil supplementation did not have substantial effects on the primary or secondary prevention of cardiovascular disease. ${ }^{2930}$ However, a meta-analysis including the most recent trials reported a significant reduction in risk of myocardial infarction. ${ }^{31}$ Further studies are needed to determine whether fish oil supplementation has a greater effect on risk of heart disease than of stroke. $^{32}$

Box 1: Population nutrient intake-definitions

- Estimated average requirement is the daily level of nutrient intake estimated to meet the requirement of half of healthy people in a population

-Inadequate nutrient intake-The population prevalence of inadequate intake is estimated as the percentage of the population with nutrient intake below the estimated average requirement

- Tolerable upper intake is the highest daily nutrient intake that is likely to pose no risk of adverse health effects to almost all healthy people in a population. As intake increases above the upper level, the potential risk of adverse effects increases.

- Excess intake-The population prevalence of excess intake is estimated as the percentage of the population with nutrient intake above the upper level

\section{Cancer}

Current evidence does not support a role of vitamin and mineral supplements in reducing cancer risk, with some evidence suggesting potential harm. $\beta$-Carotene supplementation increased the risk of lung cancer among high risk individuals in two randomised trials. The $\alpha$-Tocopherol, $\beta$-Carotene Cancer Prevention Study reported an $18 \%$ increase in relative risk among smokers randomised to $\beta$-carotene (20 mg/day) compared with those who did not. ${ }^{33}$ The $\beta$-Carotene and Retinol Efficacy Trial found that $\beta$-carotene (30 mg/day) plus vitamin A as retinol (25000 IU/day) increased risk by $28 \%$ among smokers and workers with occupational exposure to asbestos. ${ }^{34}$ The Selenium and Vitamin E Cancer Prevention Trial found that vitamin E (400 IU/day) supplementation was associated with a $17 \%$ increase in prostate cancer risk among men. ${ }^{35}$

Although maternal folic acid supplementation has been proved to reduce the risk of neural tube defects, concerns have been raised that high folic acid exposure may promote cancer progression, especially in countries with mandatory fortification. ${ }^{36}$ Most notably, folic acid supplementation at $\geq 1 \mathrm{mg} /$ day may promote the growth of undiagnosed colorectal adenomas. ${ }^{37}$ However, a meta-analysis of 11 randomised trials concluded that folic acid supplementation neither increased nor decreased site specific cancer risk within the first five years of supplementation. ${ }^{38}$

Randomised trials have failed to detect a benefit of vitamin D supplementation, alone or combined with calcium, on cancer risk at either high or low doses ${ }^{25} 39$ despite some evidence suggesting reduced total cancer mortality. ${ }^{25} 40$ The limited evidence on fish oil supplementation suggests it does not reduce cancer risk. ${ }^{2841}$

\section{Type 2 diabetes}

Current evidence does not support the use of supplements with vitamins $\mathrm{C}$ or $\mathrm{E}$, $\beta$-carotene, or fish oil to reduce the risk of type 2 diabetes, although the overall evidence from randomised trials is limited..$^{4243}$ A recent placebo controlled trial of vitamin D supplementation (4000 IU/day) failed to reduce the risk of type 2 diabetes despite significantly increasing serum 25-hydroxyvitamin D concentrations. ${ }^{44}$

\section{Osteoporosis}

Recent evidence regarding the effects of vitamin D and calcium supplementation is inconsistent. A meta-analysis of trials in community living older adults found that 


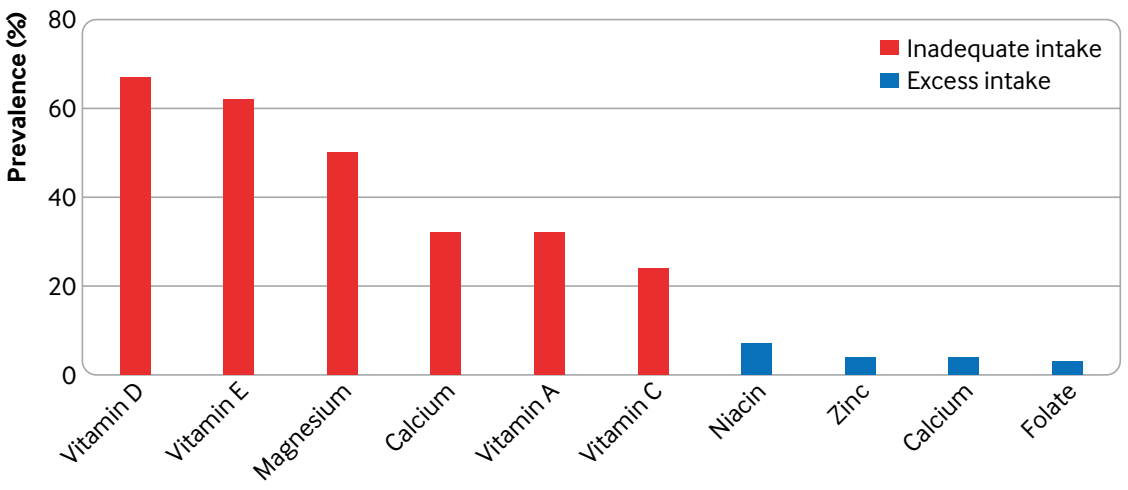

Fig 2 | Prevalence of inadequate and excess nutrient intake among US Adults, National Health and Nutrition Examination Survey 1999-2014 ${ }^{6}$

vitamin D or calcium supplementation did not reduce the risk of hip fracture or total fracture, ${ }^{45}$ whereas another meta-analysis reported that while vitamin $\mathrm{D}$ alone did not reduce fracture risk, combined calcium and vitamin D supplementation decreased the relative risk of hip fracture (16\%) and all fractures $(6 \%)$ among older adults. ${ }^{46}$ Ongoing research is assessing the effect of high dose vitamin D supplements on several health outcomes, including fractures, ${ }^{46}$ but a recent three year trial of 400, 4000, or $10000 \mathrm{IU} /$ day reported that the higher doses reduced volumetric bone density, suggesting potential for harm. ${ }^{47}$ In the absence of clear evidence on supplementation, it is prudent to ensure that dietary recommendations on calcium and vitamin D intakes are met through food and supplementation.

\section{What next?}

To date, randomised trials have largely shown no benefit of vitamin, mineral, and fish oil supplements on the risk of major non-communicable diseases in people without clinical nutritional deficiency. These results contrast with findings from observational studies, where supplemental nutrient intakes are often associated with a reduced risk of these diseases. The apparent associations from observational studies may result from unknown or unmeasured confounding factors such as socioeconomic status and lifestyle factors, including a better overall diet.

Although randomisation reduces confounding, relying exclusively on the results of randomised trials also has limitations. Trials are often conducted among high risk populations with preexisting conditions, so the findings may not be applicable to healthy individuals. Supplements may also have health benefits for population subgroups, such as people with inadequate nutrient intake from foods, but randomised trials are not usually designed to evaluate subgroup differences. Furthermore, financial and practical constraints mean that most trials are able to investigate only a single dose, which may result in selection of a dose that is either too low (no efficacy) or too high (untoward outcomes).

Nutrients obtained from foods and supplements may confer different health effects. The Cancer Prevention Study (CPS)-II Nutrition Cohort found that supplemental calcium intake at $\geq 1000 \mathrm{mg} /$ day was associated with an increased risk of all-cause mortality in men whereas high levels of calcium intake from foods had no harm. ${ }^{48}$ Among US adults in the National Health and Nutrition Examination Survey, adequate intake of nutrients from foods, but not supplements, was associated with a lower risk of all-cause mortality. ${ }^{6}$ The benefits of nutrient intake from foods may reflect synergistic interactions among multiple nutrients and other bioactive substances in foods.

The effect of supplements in specific populations warrants further investigation. Older adults are at an increased risk of malnutrition because of reduced nutrient intake and age related decreases in the bioavailability of some micronutrients. Vitamin D supplementation is recommended for breastfed infants before the introduction of whole milk and solid
- Nutrigenetics and "omics" sciences

- Personalised supplementation

- Specific needs in low and middle income countries foods. Supplements may be more effective in reducing the risk of non-communicable disease in specific ethnic groups or people with low micronutrient intake from foods. ${ }^{28}$ With a recent increase in the proportion of people reporting that they follow restricted dietary patterns such as ketogenic, Palaeolithic, vegan, and vegetarian diets, the value of supplements to meet the needs of these specific populations requires evaluation. In addition, potential nutrient-gene interactions have rarely been examined in studies of dietary supplements. Future studies on the role of nutrigenetics should help refine and personalise targeted recommendations for supplement use (box 2).

It is also important to recognise that the need for nutrient supplements is different in countries where nutrition deficiency is common. Ensuring adequate nutrition through food fortification and nutrient supplementation can be crucial to prevent serious adverse outcomes of nutrient deficiencies in low and middle income countries, especially among children $<5$ years, for whom malnutrition contributes to more than half their deaths. ${ }^{15}$

In summary, current evidence does not support recommending vitamin or fish oil supplements to reduce the risk of non-communicable diseases among populations without clinical nutritional deficiency. Continuing efforts are warranted to further understand the potentially different roles of nutrients from foods versus supplements in health promotion among a generally healthy population as well as individuals or groups with specific nutritional needs, including those living in low and middle income countries. These efforts, coupled with the integration of new research approaches, will better inform clinical practice and public health policies.

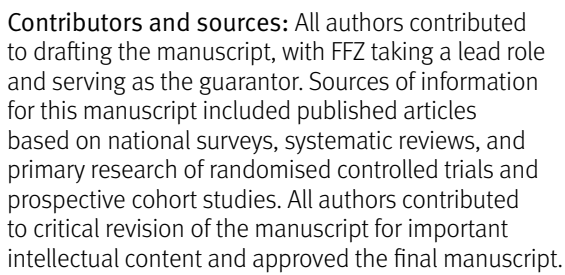

Box 2: Areas for research in vitamin and mineral supplementation

- Differing health effects of nutrients obtained from foods versus supplements

- Synergistic interactions among multiple nutrients and with other bioactive substances

- Subpopulation studies (eg, elderly people, ethnic groups, vegans) 
Competing interests: All authors have read and understood BMJ policy on declaration of interests and declared the following: FFZ declares funding from the National Institutes of Health, NIMHD (R01 MD 011501) and the Bristol Mayer Squibb Foundation (Bridging Cancer Care Programme). DL declares funding from the National Natural Science Foundation of China (NSFC 81773433) and Key Scientific Research Projects in Shandong Providence China (2017YYSP007). JBB declares funding from Danone. HM declares funding from DSM Nutritional Products, Switzerland. The funders had no role in the design or conduct of the study, collection, management, analysis, or the interpretation of the data. JBB reports service on scientific advisory boards of AdvoCare International, California Prune Board, California Walnut Commission, Church and Dwight, Cranberry Marketing Committee, Guiding Stars, Quaker Oats, Segterra, and SmartyPants, not related to this work.

Provenance and peer review: Commissioned; externally peer reviewed.

This article is part of series commissioned by The BMJ. Open access fees are paid by Swiss Re, which had no input into the commissioning or peer review of the articles. The BMJ thanks the series advisers, Nita Forouhi, Dariush Mozaffarian, and Anna Lartey for valuable advice and guiding selection of topics in the series.

Fang Fang Zhang, associate professor ${ }^{1}$

Susan I Barr, professor ${ }^{2}$

Helene McNulty, professor ${ }^{3}$

Duo Li, professor ${ }^{4}$

Jeffrey B Blumberg, professor ${ }^{1}$

${ }^{1}$ Friedman School of Nutrition Science and Policy, Tufts University, Boston, USA

${ }^{2}$ University of British Columbia, Vancouver, Canada

${ }^{3}$ Nutrition Innovation Centre for Food and Health, Ulster University, Coleraine, UK

${ }^{4}$ Institute of Nutrition and Health, Qingdao University, Qingdao, China

Correspondence to: FFZhang

fang_fang.zhang@tufts.edu

\section{(@) (D) OPEN ACCESS}

This is an Open Access article distributed in accordance with the Creative Commons Attribution Non Commercial (CC BY-NC 4.0) license, which permits others to distribute, remix, adapt, build upon this work non-commercially, and license their derivative works on different terms, provided the original work is properly cited and the use is non-commercial. See: http://creativecommons.org/ licenses/by-nc/4.0/.

\section{Check for updates}

1 Ford JA, MacLennan GS, Avenell A, Bolland M, Grey A, Witham M, RECORD Trial Group. Cardiovascular disease and vitamin D supplementation: trial analysis, systematic review, and meta-analysis. Am J Clin Nutr 2014;100:746-55. doi:10.3945/ ajcn.113.082602

2 Skeie G, Braaten T, Hjartåker A, et al. Use of dietary supplements in the European Prospective Investigation into Cancer and Nutrition calibration study. Eur J Clin Nutr 2009;63(Suppl 4):S226-38 doi:10.1038/ejcn.2009.83

3 O'Brien SK, Malacova E, Sherriff JL, Black LJ. The prevalence and predictors of dietary supplement use in the Australian population. Nutrients 2017;9:E1154. doi:10.3390/nu9101154
4 Kang M, Kim DW, Baek YJ, et al. Dietary supplement use and its effect on nutrient intake in Korean adult population in the Korea National Health and Nutrition Examination Survey IV (2007-2009) dataEur J Clin Nutr 2014;68:804-10. doi:10.1038/ejcn.2014.77

5 Black LI, Clarke TC, Barnes PM, Stussman BJ, Nahin RL. Use of complementary health approaches among children aged 4-17 years in the United States: national health interview survey, 2007-2012. Natl Health Stat Report 2015;(78):1-19.

6 Chen F, Du M, Blumberg JB, et al. Association among dietary supplement use, nutrient intake, and mortality among US adults: a cohort study. Ann Intern Med 2019;170:604-13. doi:10.7326/M18-2478

7 Kantor ED, Rehm CD, Du M, White E, Giovannucci EL. Trends in dietary supplement use among US adults from 1999-2012. JAMA 2016;316:1464-74. doi:10.1001/jama.2016.14403

8 Blumberg JB, Frei B, Fulgoni VL, Weaver CM, Zeisel SH. Contribution of dietary supplements to nutritional adequacy in various adult age groups. Nutrients 2017;9:E1325. doi:10.3390/nu9121325

9 Qato DM, Alexander GC, Guadamuz JS, Lindau ST. Prevalence of dietary supplement use in us children and adolescents, 2003-2014. JAMA Pediatr 2018;172:780-2. doi:10.1001/ jamapediatrics.2018.1008

10 Blumberg JB, Frei B, Fulgoni VL, Weaver CM, Zeisel SH. Contribution of dietary supplements to nutritional adequacy by socioeconomic subgroups in adults of the United States. Nutrients 2017;10:E4. doi:10.3390/nu10010004

11 Bailey RL, Fulgoni VL3rd, Keast DR, Dwyer JT. Dietary supplement use is associated with higher intakes of minerals from food sources. Am J Clin Nutr 2011;94:1376-81. doi:10.3945/ ajcn.111.020289

12 Harrison RA, Holt D, Pattison DJ, Elton PJ. Are those in need taking dietary supplements? A survey of 21923 adults. BrJ Nutr 2004;91:617-23. doi:10.1079/BIN20031076

13 Murphy SP, White KK, Park SY, Sharma S. Multivitamin-multimineral supplements' effect on total nutrient intake. Am J Clin Nutr 2007;85:280S4S. doi:10.1093/ajcn/85.1.280S

14 Blumberg JB, Frei BB, Fulgoni VL, Weaver CM, Zeisel SH. Impact of frequency of multi-vitamin/ multi-mineral supplement intake on nutritional adequacy and nutrient deficiencies in US adults. Nutrients 2017;9:E849. doi:10.3390/nu9080849

15 Bailey RL, West KPJr, Black RE. The epidemiology of global micronutrient deficiencies. Ann Nutr Metab 2015;66(Suppl 2):22-33. doi:10.1159/000371618

16 Bishai D, Nalubola R. The history of food fortification in the United States: its relevance for current fortification efforts in developing countries. Econ Dev Cult Change 2002;51:37-53. doi:10.1086/345361

17 Institute of Medicine Committee on Use of Dietary Reference Intakes in Nutrition Labeling. Dietary reference intakes: guiding principles for nutrition labeling and fortification. National Academies Press, 2003.

18 Shakur YA, Tarasuk V, Corey P, O'Connor DL. A comparison of micronutrient inadequacy and risk of high micronutrient intakes among vitamin and mineral supplement users and nonusers in Canada. J Nutr 2012;142:534-40. doi:10.3945/ in.111.149450

19 Briefel R, Hanson C, Fox MK, Novak T, Ziegler P. Feeding Infants and Toddlers Study: do vitamin and mineral supplements contribute to nutrient adequacy or excess among US infants and toddlers?) Am Diet Assoc 2006;106(Suppl 1):S52-65. doi:10.1016/j. jada.2005.09.041

20 Moyer VA, US Preventive Services Task Force. Vitamin D and calcium supplementation to prevent fractures in adults: U.S. Preventive Services Task Force recommendation statement. Ann Intern
Med 2013;158:691-6. doi:10.7326/0003-4819158-6-201303190-00588

21 Jenkins DJA, Spence JD, Giovannucci EL, et al. Supplemental vitamins and minerals for CVD prevention and treatment. J Am Coll Cardiol 2018;71:2570-84. doi:10.1016/j. jacc.2018.04.020

22 Martí-Carvajal AJ, Solà I, Lathyris D, Dayer M. Homocysteine-lowering interventions for preventing cardiovascular events. Cochrane Database Syst Rev 2017;8:CD006612. doi:10.1002/14651858. CD006612.pub5

23 Huo Y, Li J, Qin X, et al, CSPPT Investigators. Efficacy of folic acid therapy in primary prevention of stroke among adults with hypertension in China: the CSPPT randomized clinical trial. JAMA 2015;313:1325-35. doi:10.1001/jama.2015.2274

24 Sesso HD, Buring JE, Christen WG, et al. Vitamins E and $C$ in the prevention of cardiovascular disease in men: the Physicians' Health Study II randomized controlled trial. JAMA 2008;300:2123-33. doi:10.1001/jama.2008.600

25 Manson JE, Cook NR, Lee IM, et al, VITAL Research Group. Vitamin D supplements and prevention of cancer and cardiovascular disease. N Engl J Med 2019;380:33-44. doi:10.1056/ NEJMoa1809944

26 Hsia J, Heiss G, Ren H, et al, Women's Health Initiative Investigators. Calcium/vitamin D supplementation and cardiovascular events. Circulation 2007;115:846-54. doi:10.1161/ CIRCULATIONAHA.106.673491

27 Scragg R, Stewart AW, Waayer D, et al. Effect of monthly high-dose vitamin D supplementation on cardiovascular disease in the Vitamin D Assessment Study: a randomized clinical trial. JAMA Cardiol 2017;2:608-16. doi:10.1001/ jamacardio.2017.0175

28 Manson JE, Cook NR, Lee IM, et al, VITAL Research Group. Marine n-3 sfatty acids and prevention of cardiovascular disease and cancer. N Engl J Med 2019;380:23-32. doi:10.1056/ NEJMoa1811403

29 Abdelhamid AS, Brown TJ, Brainard JS, et al. Omega-3 fatty acids for the primary and secondary prevention of cardiovascular disease. Cochrane Database Syst Rev 2018;7:CD003177. doi:10.1002/14651858. CD003177.pub3

30 Aung T, Halsey J, Kromhout D, et al, Omega-3 Treatment Trialists' Collaboration. Associations of omega-3 fatty acid supplement use with cardiovascular disease risks: meta-analysis of 10 trials involving 77917 individuals. JAMA Cardiol 2018;3:225-34. doi:10.1001/ jamacardio.2017.5205

31 Hu Y, Hu FB, Manson JE. Marine omega-3 supplementation and cardiovascular disease: an updated meta-analysis of 13 randomized controlled trials involving 127477 participants. J Am Heart Assoc 2019;8:e013543. doi:10.1161/ JAHA.119.013543

32 Manson JE, Bassuk SS, Cook NR, et al, VITAL Research Group. Vitamin D, marine n-3 fatty acids, and primary prevention of cardiovascular disease current evidence. Circ Res 2020;126:112-28. doi:10.1161/ CIRCRESAHA.119.314541

33 Alpha-Tocopherol, Beta Carotene Cancer Prevention Study Group. The effect of vitamin $E$ and beta carotene on the incidence of lung cancer and other cancers in male smokers. N Engl J Med 1994;330:1029-35. doi:10.1056/ NEJM199404143301501

34 Omenn GS, Goodman GE, Thornquist MD, et al. Effects of a combination of beta carotene and vitamin A on lung cancer and cardiovascular disease. N Engl J Med 1996;334:1150-5. doi:10.1056/ NEJM199605023341802

35 Klein EA, Thompson IMJr, Tangen CM, et al. Vitamin $\mathrm{E}$ and the risk of prostate cancer: the 
Selenium and Vitamin E Cancer Prevention Trial (SELECT). JAMA 2011;306:1549-56. doi:10.1001/ jama.2011.1437

36 Mason JB. Folate, cancer risk, and the Greek god, Proteus: a tale of two chameleons. Nutr $\operatorname{Rev} 2009 ; 67: 206-12$. doi:10.1111/j.17534887.2009.00190.x

37 Cole BF, Baron JA, Sandler RS, et al, Polyp Prevention Study Group. Folic acid for the prevention of colorectal adenomas: a randomized clinical trial. JAMA 2007;297:2351-9. doi:10.1001/ jama.297.21.2351

38 Vollset SE, Clarke R, Lewington S, et al, B-Vitamin Treatment Trialists' Collaboration. Effects of folic acid supplementation on overall and site-specific cancer incidence during the randomised trials: meta-analyses of data on 50,000 individuals. Lancet 2013;381:102936. doi:10.1016/S0140-6736(12)62001-7

39 Lappe J, Watson P, Travers-Gustafson D, et al. Effect of vitamin $D$ and calcium supplementation on cancer incidence in older women: a randomized clinical trial. JAMA 2017;317:1234-43. doi:10.1001/ jama.2017.2115

40 Manson JE, Bassuk SS, Buring JE, VITAL Research Group. Principal results of the vitamin $d$ and omega-3 trial (VITAL) and updated meta-analyses of relevant vitamin D trials. / Steroid Biochem Mol Biol 2020;198:105522. doi:10.1016/j. jsbmb.2019.105522

41 Song M, Lee IM, Manson JE, et al, VITAL Research Group. Effect of supplementation with marine $\omega-3$ fatty acid on risk of colorectal adenomas and serrated polyps in the US general population: a prespecified ancillary study of a randomized clinical trial. JAMA Oncol 2019;6:108-15. doi:10.1001/ jamaoncol.2019.4587

42 Seida JC, Mitri J, Colmers IN, et al. Clinical review: Effect of vitamin D3 supplementation on improving glucose homeostasis and preventing diabetes: a systematic review and meta-analysis. / Clin Endocrinol Metab 2014;99:3551-60. doi:10.1210/jc.2014-2136

43 Song Y, Cook NR, Albert CM, Van Denburgh M, Manson JE. Effects of vitamins $C$ and $E$ and betacarotene on the risk of type 2 diabetes in women at high risk of cardiovascular disease: a randomized controlled trial. Am J Clin Nutr 2009;90:429-37. doi:10.3945/ajcn.2009.27491

44 Pittas AG, Dawson-Hughes B, Sheehan P, et al, D2d Research Group. Vitamin D supplementation and prevention of type 2 diabetes. $N$ Engl J Med 2019:381:520-30. doi:10.1056/ NEJMoa1900906
45 Zhao JG, Zeng XT, Wang J, Liu L. Association between calcium or vitamin $\mathrm{D}$ supplementation and fracture incidence in community-dwelling older adults: a systematic review and metaanalysis. JAMA 2017;318:2466-82. doi:10.1001/ jama.2017.19344

46 Yao P, Bennett D, Mafham M, et al. Vitamin D and calcium for the prevention of fracture: $\mathrm{a}$ systematic review and meta-analysis. JAMA Netw Open 2019;2:e1917789. doi:10.1001/ jamanetworkopen.2019.17789

47 Burt LA, Billington EO, Rose MS, Raymond DA Hanley DA, Boyd SK. Effect of high-dose vitamin D supplementation on volumetric bone density and bone strength: a randomized clinical trial. JAMA 2019;322:736-45. doi:10.1001/ jama.2019.11889

48 Yang B, Campbell PT, Gapstur SM, et al. Calcium intake and mortality from all causes, cancer, and cardiovascular disease: the Cance Prevention Study II Nutrition Cohort. Am J Clin Nutr 2016;103:886-94. doi:10.3945/ ajcn.115.117994

Cite this as: $B M / 2020 ; 369: \mathrm{m} 2511$

http://dx.doi.org/10.1136/bmj.m2511 